\title{
Microneedles for Drug Delivery
}

\author{
Gary W. Cleary
}

Received: 15 October 2010 / Accepted: 15 October 2010 / Published online: 17 November 2010

(C) Springer Science+Business Media, LLG 2010

\section{INTRODUCTION}

The theme section highlighted in this issue of Pharmaceutical Research provides a snapshot of the latest information on today's microneedle research and development, predominately in transdermal applications. Fifteen original papers from academia and industry cover research and development of microneedles from concept to human clinical studies and everything in between. The research presented here is diverse in the use of various materials used to make microneedles and in the molecules that can be delivered, from small molecules that generally cannot permeate intact skin enough to reach therapeutic blood levels, to large molecules, including vaccines, proteins, and polypeptides. These studies also show a fundamentally different approach to today's injection methodology of syringes and needles. Drug molecules inserted into the skin via microneedles cause no pain and no bleeding and allow for delivering drugs from dry dissolvable microneedles rather than liquids. After use and during disposal of microneedles, particularly the dissolvable ones, there are no accidental needle sticks and fewer issues with biohazardous waste.

\section{THE BEGINNING}

Transdermal microneedles, as a whole, are now beginning to emerge as a credible way to deliver large and small drug

G. W. Cleary $(\bowtie)$

Corium International Inc.

Menlo Park, CA, USA

e-mail: gary@coriumtech.com molecules through the skin. The journey to this point in development has taken nearly forty years. The first concept to make micropores in the skin came in the form of a U.S. patent by Gerstel and Place from Alza Research in the early 1970s (1). Unfortunately, the technology to make and market arrays of micron-sized needles economically was not available then. Microchip fabrication technology took about 25 years to converge with newer ways to make longer three-dimensional microstructures of silicon, resuscitating the possibilities for mass production of microneedle arrays $(2,3)$. With the onset of new microfabrication techniques available in the microchip industry, such as the LIGA method, came various unique, three-dimensional designs with greater aspect ratios of solid and hollow microneedles where the microstructure was in plane (i.e. parallel to the base of the microneedle backing) and out of plane (i.e. perpendicular to the base of the microneedle backing). Ultimately, out-of-plane microneedles have become more popular because production of structural arrays was simpler than hollow in-plane designs $(2-5)$.

\section{DESIGN, CHARACTERIZATION, MATERIALS, AND MICROFABRICATION}

The mid to late 1990s could be considered the starting point for today's work in microneedles. The focus at that time was on finding ways to microfabricate the microstructures that make up a three-dimensional array of microneedles from various materials. Early on, silicon and metal were used, based on the microchip technology approach of microfabrication. Now, papers in this theme issue describe designs of solid and hollow microneedles with 
various microstructures, different designs and materials, and several microfabrication approaches (Fukushima et al., Wendorf et al., Burton et al., Donnelly et al., Andrianov et al.). Two papers describe the fabrication of various microneedles from different materials using various micromoulding processes or other methods, such as lasers (Donnelly et al., Coulman et al.). Microneedles were then characterized in vivo on humans, comparing the microstructure design, height, and other parameters of water-soluble and -insoluble polymers and steel. Microneedles were inserted into human skin in vivo and observed using new imaging methodologies. Each paper in this theme issue helps to show the degree of advancement that has been accomplished in the past five years or so with various materials, designs, and microfabrication methods.

\section{FUNDAMENTALLY DIFFERENT APPROACH FROM CONVENTIONAL INJECTIONS}

Miniaturization and the use of solid microstructures and hollow needles with a syringe take a totally different approach to delivering drugs through the skin. From early on, human studies have shown that microneedles ranging from 200 to 750 microns in length are painless and bloodless (5). The micropores in the skin that are created by the microneedles take from four to eight hours to reach closure (i.e., reseal) if the micropores are left without any covering (non-occluded) or about sixteen to twenty-two hours for complete closure when the micropores are covered (occluded) (6). Microneedles have a dimensional size difference ranging from 100 to 1000 times smaller than the conventional long centimeter-sized stainless steel needles that require a syringe. With this smaller micro-sized needle, less pain and bleeding are observed. Three papers in this theme section revisit this phenomena when inserting microneedles into skin and study the time for closure of the micropores using new imaging techniques ex vivo and in vivo (Coulman et al., Kalluri \& Banga, Donnelly et al.). Another paper presents an exploratory panel study of human subjects and healthcare providers in focus groups to determine qualitative views on microneedles (Birchall et al.).

\section{ACCESS TO NEW IMAGING TOOLS}

This theme section also shows the accessibility of using existing or recently developed imaging equipment, such as Optical Coherence Tomography and several fluorescence techniques, to see microneedles or microparticles following insertion into skin in vivo, in vitro, or ex vivo (Donnelly et al., Coulman et al., Kalluri \& Banga).

\section{ENLARGING THE UNIVERSE OF DRUGS: SMALL AND LARGE ACTIVE MOLECULES}

Several papers presented here clearly illustrate that microneedles are in an early stage with proof-of-principal, in vivo pharmacokinetic studies in animals. These studies show the capability to deliver a wide range of small and large therapeutic molecules ranging from Naloxone (327 Da) to Etanercept (Enbrel®) (132 kDa) through the skin using hollow microneedles (Burton et al., Harvey et al.). Other investigators have studied similar-sized molecules with the tips of individual solid microneedles coated with a dissolvable drug coating or uncoated and used as a pretreatment method. The microneedles were then inserted in the animal to release the drug from the coated tip or to produce micropores followed by a passive transdermal patch that delivered drugs such as L-carnitine (161 Da), naltrexone (341 Da) and theophylline (180 Da) through the skin (Zhang et al., Milewski \& Stinchcomb, Donnelly et al.). Another type of drug delivery uses polymeric dissolvable microneedles to deliver large-sized drug molecules, such as human growth hormone $(22 \mathrm{kDa})$ and demopressin $(1.1 \mathrm{kDa})$ through the skin (Fukushima et al., Donnelly et al.).

\section{VACCINES}

Another opportunity for microneedles that lies ahead is the delivery of vaccines. Four interesting papers here cover four different aspects of delivering vaccines with or without an adjuvant using solid-state microneedles. One important attribute of solid microneedles is that they allow the vaccine and/or the adjuvant to be in the solid phase rather than suspended in water for injection by syringe and needle. Here, an initial study shows that the stability of inactivated influenza maintains its immunogenicity for up to a month at $25^{\circ} \mathrm{C}$ with trehalose compared to zero activity without trehalose. The investigators suggested additional work could further lead to longer stability times (Kim et al.). In another study, a team of investigators using a pretreatment to form micropores followed by topically applying diphtheria toxoid in a cationic liposome with cholera as an adjuvant provided immunogenicity, while anionic vesicles carrying diphtheria toxoid with cholera as an adjuvant did not have any immunogenicity. Here, the formulation was key to maintain immunogenicity (Ding et al.). In another paper, a solid microneedle made of metal and dip-coated 
with a polyphosphazene-based adjuvant provided improved stability of the protein during microfabrication and modulated the release of the protein (TITC-BSA) compared to a liquid solution formulation (Andrianov et al.). Finally, another team using a biodegradable microneedle embedded with $10 \mu \mathrm{g}$ recombinant protective antigen (rPA, $83 \mathrm{kDa}$ ) was able to show in rats that the biodegradable microneedles delivered rPA intradermally and had equivalent or higher immune response, based on IgG EISA titer, than $10 \mu \mathrm{g}$ of iPA delivered intramuscularly or intradermally using a conventional syringe and needle (Wendorf et al.).

\section{HUMAN CLINICAL STUDIES}

Microneedle development has advanced now into human clinical studies that fall under the scrutiny of FDA regulations. In 2009, a six-month, randomized, multicenter, blinded, multi-dose, Phase 2 clinical study using microneedles to deliver recombinant human parathyroid hormone 1-34, teriparatide (PTH, $4.1 \mathrm{kDa}$ ) offered the first results of this advancement in microneedles (7). The study enrolled 165 patients between 50 and 81 years of age with severe osteoporosis and was conducted at thirteen sites across North and South America. It showed that a $40 \mathrm{mcg}$ of PTH delivered using solid titanium microneedles delivered an effective amount of PTH, comparable to a $20 \mathrm{mcg}$ Forteo (PTH) injection. The same study also demonstrated that the microneedle system increased the total hip Bone Mineral Density by $+1.33 \%$ compared to Forteo at $0.094 \%$ and a placebo at a decrease of $-0.634 \%$.

One paper in this theme section describes the delivery of the same recombinant human parathyroid hormone 1-34, teriparatide (PTH, $4.1 \mathrm{kDa})$, as part of a Phase 2 study using the same microneedle system as discussed earlier: solid titanium microneedles with the sharp tips coated with a formulated PTH using a dip process (7). The microneedle patch is applied to the skin using an applicator. These investigators show 94-98\% stability for two years at $25^{\circ} \mathrm{C}$ and six months at $40^{\circ} \mathrm{C}$. The pharmacokinetic data was taken from both the PTH delivered by the microneedles and comparator, which was a conventional injectable pen that contained rhPTH $\left(\right.$ Forteo ${ }^{\circledR}$, Eli Lilly, Co.). The PTH from the microneedle patch showed a more rapid onset of delivery and a $\mathrm{C}_{\max }$ that peaked earlier than the Forteo using a subcutaneous injection pen. The microneedle patch's pharmacokinetic profile was consistent at the beginning and end of the Phase 2 study. The patch also showed gains in bone density at the hip and lumbar spine sites (Daddona et al.).

\section{MICRONEEDLE DRUG DELIVERY FOR USE IN OCULAR DRUG DELIVERY}

One paper used microneedles to treat the eye ex vivo (Patel et al.). Here, the investigators explored use of a single hollow microneedle that was slightly longer $(800-1000 \mu \mathrm{m})$ than most microneedles used transdermally for skin delivery. The investigators inserted the microneedle into the eye's sclera and infused a suspension of nanoparticles further into the eye until they reached the suprachoroidal space. Using microscopy, it was then determined through imagery that the microneedle reached the targeted space.

Delivery to the back of the eye presents a challenge for delivery systems in general. However, microneedle technology may someday find its place in opthalmology on a regular basis as well as other fields of drug delivery beyond transdermal and ocular delivery.

\section{CONCLUSION}

Although there are challenges still ahead for this newcomer to the field of traditional drug delivery systems, the results found in the various papers presented in this Microneedles for Drug Delivery theme section of Pharmaceutical Research provide great optimism for the future of microneedles. The human data and pharmaceutical research and development presented here offer only a glimpse at the early emerging stages of the products of the future. Microneedles have shown here and in many other publications that they can serve as a suitable alternative to conventional injection delivery systems of small active ingredients as well as the larger molecular weight proteins, peptides, and vaccines.

\section{INTERVIEW WITH DR. GARY W. CLEARY}

\section{What do you think holds the key to your success as a pharmaceutical scientist?}

Curiosity, persistence, flexibility, and sustainability in the areas of skin biology, dermatology, and transdermal-based pharmaceutics over many years have been the keys to my success. Also, lifetime learning is important to me and helps me keep abreast with new scientific findings and approaches to potential new and exciting technologies. This is exemplified by my participation in passive and active transdermal technologies over the past thirty-five years when these technologies were in their infancy all the way to today's microneedles and other active transdermal delivery systems. 
What do you consider to be your key research accomplishments?

My key research accomplishments are really based on having an array of top multidisciplinary teams of scientists and engineers with the appropriate education skill sets along with the same passion to tackle all the experimental work and developmental activities that are needed to develop transdermal products. A fundamental understanding of areas in basic research through applied research followed by engineering, chemistry, biology, pharmacokinetics, and many other backgrounds makes it all happen in the early stages of development. No one individual has all the information to be successful. The success lies with the team. I've seen this phenomenon happen over and over in my career while working in start-up companies such as Alza, Key Pharmaceuticals, Genentech, Cygnus, and Corium. Many useful first-of-their-kind products have come out of those companies.

\section{What was the turning point in your career?}

The turning point came when I first realized, or at least felt, that I had the technical and mental capacity to start up a new company. Having spent most of my career in the early phases of start-up companies compounded by innovative ideas and usually blank sheets of paper helped me build up the confidence to start Cygnus and Corium, as a founder and co-founder, respectively.

\section{Who has most influenced your research career?}

Aside from having friends and family that encouraged me to have a career in research and development, there were others from the companies where I worked or from schools I attended. There were professors like Sidney Riegelman at UCSF, who was inspirational in his classroom when he brought mathematics, pharmacology, physiology, biochemistry, anatomy, and chemistry all rolled together into a course called "pharmacokinetics" before there were many books written about pharmacokinetics in the 1960s. Pharmacokinetics pulls several skill sets together, and I might say here is where I was first exposed to multidisciplinary subjects.

Joel Zatz at Rutgers was my major professor and advisor on my Ph.D. thesis, which was basically in surface chemistry. Surface chemistry has been with me all these years, enabling me to understand adhesives issues with transdermal development and, more recently, nanotechnology. I would have to say I was very influenced by most of the professors I studied under while getting my Pharm.D, Ph.D., and MBA. The MBA was quite helpful in crossing the bridge from technology to operational aspects of companies and industry. Also while at Rutgers studying for my doctorate degree, I spent three summers working in the Basic Research Group at Johnson \& Johnson in New Brunswick, NJ. It was there that I had a strong dose of what it was like to work in an industrial setting. In industry, I still needed to have well-thought-out experimental designs, which I learned in basic research at J\&J. I also enjoyed working in areas that needed a multidisciplinary approach.

I found inspiration in the founders of companies where I worked, such as Alejandro Zaffaroni at Alza, Mike Jaharis at Key Pharmaceutical, and Bob Swanson at Genentech, who provided the opportunity to develop the vision, courage, and tenacity needed to tackle the unseemly large numbers of hurdles required to jumpstart a new company and survive in a pharmaceutical/biotech-based start-up company.

Of course, without my scientific colleagues and my family, who believed in me to try some far-fetched ideas from time to time, I wouldn't be here to answer these questions.

\section{Pharmaceutical scientists are faced with the dilemma} of having to publish in biomedical or basic science journals. Does this mean cutting-edge science will not likely be featured in journals like Pharmaceutical Research?

I believe there is great opportunity to feature cutting-edge science articles from pharmaceutical scientists in journals like Pharmaceutical Research. For example, very early discoveries and understandings in systems biology are just the beginning of a continuum of processes that ultimately leads to a therapeutic product. Once a cutting-edge concept materializes, a battery of life sciences and physical sciences have to come together to begin to apply the necessary medical and pharmaceutical sciences, bioengineering, chemistry, and imaging tools that may need to develop in order to bring the concept to a therapeutic reality. This then becomes cutting-edge work to advance, say, product development, clinical study strategies, specialized manufacturing equipment to make first-of-their-kind products, and so on. Throughout this continuum of basic research, applied research, translational medicine, drug delivery, clinical studies, and analytical methods, regulatory requirements are taking place. From concept to commercialization, Pharmaceutical Research and AAPS play an important role. One might instead say that basic natural scientists and physical scientists are faced with the dilemma of having to publish in journals like Pharmaceutical Research, since the science behind the cutting-edge concept needs to quickly interface with the science, medicine, and engineering 
community found in AAPS. Perhaps AAPS would consider publishing a number of journals related to cutting-edge sciences such as systems biology (stem cells), chemistry (nanoparticles), and drug delivery (targeted nanoparticles, scaffolds, etc.), for example.

Where is the field of Microneedles for Drug Delivery going, and how do the articles in this theme section fill the gap?

The field of Microneedles for Drug Delivery has a bright future ahead of it. With large molecules reaching the marketplace, microneedles will extend beyond the passive transdermal patch capabilities and eliminate some use of the syringe-and-needle approaches. Microneedles have come a long way since the first patent issued in 1976. Materials and manufacturing methods arrived in the 1990s and, in the past decade, have been tested in human studies. This theme section brings us up to date on where microneedles are today and provides a look into the future. It provides proof-of-principle in delivery of large molecules (proteins, peptides, vaccines) and small molecules needing higher blood levels than can be reached by passive transdermals. Causing little or no pain or bleeding when used properly, microneedles will find great acceptability among patients. Micropores made by microneedle arrays have been shown to close and reseal within twenty-four hours and as fast as a couple of hours. Their formulas can deliver solids and liquids through the skin. Though we have come a long way since the 1970s, we're still only at the beginning with respect to microneedles.

\section{What are the challenges for designing and using novel microneedles for transdermal drug delivery, and how can these challenges be overcome for enhanced drug absorption through the skin?}

There will be challenges coming from a number of directions. Selection of materials is important for these microneedle products. The materials will impact biocompatibility and must have the appropriate mechanical properties for fabrication and ultimate use. The cost of materials and the microneedle product will need to be scaled up to large quantities and reproducible from batch to batch at a cost that will be acceptable to reimbursement agencies and companies. There is already evidence that microneedles can achieve blood levels that are equal to or perhaps better than the traditional methods of drug administration. This new method of penetrating the skin with no pain and no bleeding is user friendly. Physicians must understand that microneedles are easier to dispense and dispose of than syringes and needles. The papers in this theme section touch on a lot of these challenges. There is still a bit of time to go before microneedle products sweep the marketplace.

\section{What is the key to developing successful collaborative relationships, and how can your company help?}

What I find as keys to successful collaboration are communication and building relationships. Relationships are important within various levels of people in groups, as well as between levels or departments within an organization and between different organizations. It is important to establish good relationships up and down the ranks in both academia and industry, and relationships between the two realms are also very important. There are common interests for all of these groups, and they should strive to maintain good communications and common understandings.

\section{What is your philosophy of providing internship or curricular practical training for graduate students in pharmaceutical companies?}

At my previous company Cygnus and now at Corium we have had a range of students who interned for a few months. At Cygnus, students performed research for their theses. I believe it's important for students to have some exposure to the industrial side or research and development at some point in their education. However, I also believe that a mentor should be available at the company who can spend time with the student. In the very early stage of a start-up it is very difficult for scientists to multitask to get their daily work done. However, it is always nice to have a fully experienced mentor-like scientist/ engineer who likes working with the students while they are at the industrial site. We also have had many intern students become employees of the company, which also has worked well.

\section{What challenges face the pharmaceutical sciences?}

The sciences and technologies are continually advancing with new and changing discoveries, creating exciting opportunities to contribute to advancing health and quality of life. Today, pharmaceutical sciences encompass a large breadth of many sciences that lead to many technologies. It now takes many disciplines collaborating to cover the necessary research and development needed to evolve the new sciences and technologies. The challenge that pharmaceutical sciences face is to keep up with the new technologies that derive from outside their sphere of influence. As I look back over the years, it appears that changes are constant in our field. There are 
newer tools that help us reach solutions faster that were not available in earlier times. I stopped using my slide rule years ago. Now, look at the size of laptops which can do computation that once used the same space as a large house. It seems like technology and tools for technologies are moving faster as I'm getting older. Perhaps, I'm just getting slower.

\section{What are your views for collaboration between industries and universities?}

I believe that the collaboration should not only take place but that it is essential for individuals, universities, and industries all to continue to do what they do best. Universities should continue to do basic research along with education. Industry should fully understand the needs of universities and vice versa. What seems to be happening is a convergence of industries and universities to some extent. As long as there is higher education available for individuals somewhere, then perhaps convergence shouldn't be a problem.

\section{REFERENCES}

1. Gerstel MS, Place VA (1976) Drug Delivery Device, US Patent No.3,964,482.

2. Lin L, Pisano A, Muller R (1993) Silicon processed microneedles, in Dig. Transducer '93, Intl. Conf. Solid-State Sensors and Actuators, 237-40.

3. Cormier M, Theeuwes F (1997) WO 97/4811, EU Published Patent Application.

4. Henry S, McAllister D, Allen M, Prausnitz M. Microfabricated microneedles: A novel approach to transdermal drug delivery. J Pharm Sci. 1998;87:922-5.

5. Gill HS. Effect of microneedle design on pain in human volunteers. Clin J Pain. 2008;7:585-94.

6. Gupta J, Andrews S, Gill HS, Prauznitz MR. Kinetics of skin resealing after insertion of microneedles in human subjects, Abstract 2008 3348, The 35th Annual Meeting and Exposition of the Controlled Release Society, July 19-22, 2008.

7. Cosman F, Lane NE, Bolognese MA, Zanchetta JR, GarciaHernandez PA, Sees K, et al. Effect of Transdermal Teriparatide Administration on Bone Mineral Density in Postmenopausal Women. J Clin Endocrinol Metab. 2009;95:151-8.

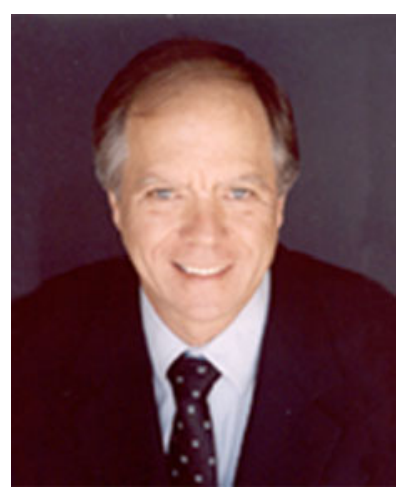

Dr. Gary W. Gleary is an internationally recognized scientist in the field of drug delivery systems and polymer technologies as well as a Founder of Cygnus, Inc. and co-founder of Corium International, Inc. He has served as President and Chief Technology Officer of Corium International, Inc. and Chairman, President, and Chief Technical Officer of Cygnus, Inc. Corium (www.coriumgroup.com) specializes in dermal, transdermal, mucosal, and transmucosal drug delivery platforms and manufacturing systems, with a special emphasis on Active Transdermal Systems, MicroCor ${ }^{\circledR}$ (a microneedle technology), MicroJet ${ }^{\mathrm{TM}}$ (a pulsed liquid jet technology), and Corplex ${ }^{\mathrm{TM}}$ (a polymer technology).

Dr. Cleary holds more than twenty-seven issued U.S. patents, with more than twenty-five other U.S. patents pending related to transdermal, mucosal, polymer, and other drug delivery technologies. He has received the Entrepreneur of the Year Award in Life Sciences for Northern California, the Biomaterials Entrepreneur Award of the New Jersey Center for Biomaterials, the AAPS Rainer Hoffman Award for Product Through Science, and the Distinguished Alumnus of the Year Award from University of California, San Francisco (UCSF). This year, Corium received the Edison Award for the MicroJet Technology and the P\&G Partner of the Year Award.

Dr. Cleary earned a Pharm.D. from UCSF, Ph.D. in Pharmaceutical Sciences from Rutgers University, and MBA in Health Sciences from the University of Miami. He has held research and management positions at the USPHS/FDA, Alza, Key Pharmaceuticals, Genentech, and Cygnus. He has worked on projects that have been the first-oftheir-kind passive TDS products (scopolamine, clonidine, nicotine, norelgestromin/ethinyl estradiol). At Cygnus, he explored ultrasound, iontophoresis, and electroporation active transdermal technologies throughout the 1980s and 1990s. Cygnus received FDA approval of the GlucoWatch, which was the first "active programmable transdermallike" product to reach the market that used microprocessors, microchips, LCD, biosensor, and an enzyme with iontophoresis technology.

Dr. Cleary is a Fellow of CRS, American Association Pharmaceutical Scientists (AAPS), and American Institute of Medical Bioengineering (AIMBE), and Past President Controlled Release Society (CRS), and member of several other professional affiliations. He currently serves as CRS Chair College of Fellows and is on the Advisory Board at the University of Pacific, the Board of Directors at Corium, the Board of the Community School of Music and Arts in Mt. View CA, and the Scientific Advisory Board of Appian Labs. His past board affiliations include Angiogenix, Cygnus, NJ Center for Biomaterials, Center for Military Biomaterials Research, Purdue University Bioengineering, Rutgers Biomedical Engineering, and UCSF School of Pharmacy. 